\title{
Structural and Functional Analysis of FLAG Tagged-Subunit 8 of Yeast Saccharomyces cerevisiae Mitochondrial ATPSynthase
}

\author{
I MADE ARTIKA \\ Department of Biochemistry, Institut Pertanian Bogor, Jalan Raya Pajajaran, Bogor 16144, Indonesia \\ Phone/Fax: +62-251-323166, E-mail: imart171@yahoo.com
}

\begin{abstract}
Yeast mitochondrial ATP synthase is a multisubunit complex composed of at least 17 different subunits. Subunit 8 of yeast mitochondrial ATP synthase is a hydrophobic protein of 48 amino acids encoded by the mitochondrial ATP8 gene. Although ATP synthase from eukaryotes and prokaryotes shows a similar basic structure, no homologue of subunit 8 is found in prokaryotes such as Escherichia coli. Subunit 8 has three distinct domains; an $\mathrm{N}$-terminal domain, a central hydrophobic domain and a $\mathrm{C}$-terminal domain. In order to elucidate its structure and function, a set of nuclear genes encoding subunit 8 variants was designed to incorporate a FLAG tag at the $\mathrm{C}$-terminus and a mitochondrial signal peptide at the $\mathrm{N}$-terminus. Each gene was cloned into a yeast expression vector and then allotopically expressed in a yeast strain lacking endogenous subunit 8 . Structural and functional analysis showed that the hydrophobic character of the central hydrophobic domain of subunit 8 is critical for the ATP synthase function. Subunit 8 is sensitive to charge manipulation at the $C$-terminus. The positively charged residues at the $\mathrm{C}$-terminal domain are important for subunit 8 assembly and hence its function.
\end{abstract}

Key words: allotopic expression, ATP synthase, mitochondria, yeast

Mitochondrial ATP synthase (E.C.3.6.1.3) also known as $\mathrm{F}_{0} \mathrm{~F}_{1}$-ATPase, is key enzyme which plays a major role in the formation of ATP used to drive cellular processes. The yeast mitochondrial ATP synthase is a multisubunit complex composed of at least 17 subunits grouped into two sectors, viz. $\mathrm{F}_{1}$ and $\mathrm{F}_{0}$ sectors. The $\mathrm{F}_{1}$ sector lies at the inner (matrix) surface of the inner mitochondrial membrane and is comprised of subunits $\alpha, \beta, \gamma, \delta$, and $\varepsilon$, with a stoichiometry of $3: 3: 1: 1: 1$, all of which are encoded at the nucleus (Cox et al. 1992). The $\mathrm{F}_{0}$ sector spans the membrane and is composed of subunits b, OSCP, d, e, f, g, h, i/j, k which are encoded by nuclear genes, and subunits 6,8 , and 9 , which are encoded by mitochondrial genes (Stephens et al. 2000). In the inner mitochondrial membrane, the ATP synthase complex can form a dimer (Fronzes et al. 2006).

Subunit 8 of yeast mitochondrial ATP synthase is a small hydrophobic polypeptide of 48 amino acids encoded by the ATP8 gene (Macreadie et al. 1983). Analysis of its primary structure has led to the identification of three distinct domains; e.g., an N-terminal domain, a central hydrophobic domain (CHD), and a C-terminal domain. The CHD which spans residues 14 to 32 , has been predicted to act as a transmembrane stem (Nagley et al. 1990). As a mitochondrially encoded protein, subunit 8 is transcribed, translated, and transported within the organelle. Subunit 8 is present in eukaryotic ATP synthases but not in prokaryotic enzyme complexes (Cox et al. 1992). This means that bacterial ATP synthase naturally functions without the existance of subunit 8 . The immediate question therefore is how this subunit functions in the enzyme complex. Detailed analysis of the subunit 8 structure and function is still lacking. Although subunit 8 has been considered to participate in proton translocation (Nagley et al. 1988), the exact role of subunit 8 in this function remains unclear.

In order to elucidate its structure and function, an allotopic expression system for subunit 8 has been developed. Allotopic expression is the deliberate relocation of organellar genes to the nucleus and delivery of the gene products from the cytoplasm to the corresponding organelle. For allotopic expression of subunit 8 , a nuclear version of subunit 8 gene to be expressed in the nucleocytosolic system has been designed. To ensure that the cytoplasmically synthesized subunit 8 was imported into mitochondria, sequences encoding a mitochondrial signal peptide were fused to the N-terminus of the gene (Gearing et al. 1985). The allotopic expression system has been applied to study various aspects of the subunit 8 molecular biology. This system has also been successfully used to express FLAG tagged-subunit 8 protein (Artika 2006). The allotopically expressed FLAG tagged-subunit 8 protein was imported into mitochondria and assembled into a functional ATP synthase complex. The main purpose of FLAG tag addition to subunit 8 protein was to allow the use of immunochemical methods to detect subunit 8 protein. However, since the FLAG tag is highly charged, its incorporation into C-terminus region of subunit 8 changes the nature, number, and distribution of the charged residues that may affect the structure and functioning of subunit 8 . In the present study a set of subunit 8 variants containing either double negative charges or double positive charges within the CHD was FLAG tagged and then allotopically expressed in a mutant yeast strain lacking endogenous subunit 8 . Structural and functional consequences of the introduction of FLAG tag residues to subunit 8 variants are discussed.

\section{MATERIALS AND METHODS}

Materials. Saccharomyces cerevisiae strain M31 [atp8, mit $^{-}$, his6, ade1], a collection strain of the Department of Biochemistry and Molecular Biology, Monash University, has previously been described (Nagley et al. 1988). Strain YM2 is strain M31 expressing non-tagged-subunit 8 gene fused with a mitochondrial signal peptide (Roucou et al. 1999). The set of gene constructs encoding subunit 8 variants 
fused with a mitochondrial signal peptide has been described previously (Papakonstantinou et al. 1996). The yeast expression vector pPD72 used for allotopic expression has been described (Law \& Devenish 1988). The vector has the yeast $A D E 1, L E U 2$, and $U R A 3$ genes as selectable markers.

Gene Modification and Molecular Cloning. The FLAG epitope tag was incorporate into each variant by using a PCR-based mutagenesis technique (Artika 2006). The primers used were designed to incorporate additional nucleotide sequences encoding hexapeptide (DYKDDD) representing the FLAG epitope tag at the C terminus of the gene with two serine residues functioning as a bridge between the subunit 8 and the FLAG tag. Cloning of the gene constructs into the yeast expression vector was carried out using standard methods (Sambrook et al. 1989).

Yeast Transformation. Introduction of recombinant plasmid vector into yeast strain M31 was done as described by Klebe et al. (1983).

Determination of Generation Time. Generation time of each strain was determined as described by Gray et al. (1996).

Isolation of Mitochondria. Intact mitochondria were prepared using the glass bead method (Lang et al. 1977). The protein concentration of isolated and washed mitochondria was determined using the Bio-Rad protein micro-assay procedure based on the method of Bradford (1976).

Protein Analysis. SDS polyacrylamide gel electrophoresis (SDS-PAGE) was performed as described by Laemmli (1970) using a dual adjustable slab gel unit. Following SDS-PAGE, the proteins were transferred onto an immobile-P membrane (PVDF). The membrane was then incubated overnight at $4{ }^{\circ} \mathrm{C}$ in blotting solution containing primary antibody. After washing away the unbound primary antibody, the membrane was incubated for 1 hour at room temperature in blotting solution containing secondary antibody (Alkaline Phosphatase Conjugate). Proteins were visualized using a Vistra Alkaline Phosphatase Conjugate Substrate Kit (Amersham Life Science, Bucks, U.K.).

\section{RESULTS}

FLAG Tagged-Subunit 8 Variants Assembled into Functional Mitochondrial ATP Synthase Complexes. The addition of nucleotide sequences encoding the FLAG epitope tag to the $\mathrm{C}$-terminus of six different subunit 8 variants resulted in six FLAG tagged-subunit 8 variants as shown in Table 1.

In order to examine their functionality in vivo, the genes encoding the FLAG tagged-subunit 8 variants fused with mitochondrial signal peptide were cloned into a yeast

Table 1 FLAG epitope tagged-subunit 8 variants of yeast mitochondrial ATP synthase

\begin{tabular}{cl}
\hline Variant/strain & \multicolumn{1}{c}{ Mutation } \\
\hline FTC2 & Wildtype, 49S, 50S, 51D, 52Y, 53K, 54D, 55D, 56D \\
DF66 & G16 $\rightarrow$ D, F17 $\rightarrow$ D, 49S, 50S, 51D, 52Y, 53K, 54D, 55D, 56D \\
DF67 & G16 $\rightarrow$ R, F17 $\rightarrow$ R, 49S, 50S, 51D, 52Y, 53K, 54D, 55D, 56D \\
DF68 & L23 $\rightarrow$ D, L24 $\rightarrow$ D, 49S, 50S, 51D, 52Y, 53K, 54D, 55D, 56D \\
DF69 & L23 $\rightarrow$ R, L24 $\rightarrow$ R, 49S, 50S, 51D, 52Y, 53K, 54D, 55D, 56D \\
DF70 & Q29 $\rightarrow$ D, F30 $\rightarrow$ D, 49S, 50S, 51D, 52Y, 53K, 54D, 55D, 56D \\
DF71 & Q29 $\rightarrow$ R, F30 $\rightarrow$ R, 49S, 50S, 51D, 52Y, 53K, 54D, 55D, 56D \\
\hline
\end{tabular}

expression vector pPD72. The resultant recombinant plasmids were then allotopically expressed in yeast cells strain M31. The strain M31 lacks of endogenous subunit 8 due to mutation in the ATP 8 gene. Since subunit 8 is an essential subunit of the mitochondrial ATP synthase complex, the strain M31 is therefore unable to form functional mitochondrial ATP synthase complex. Consequently, strain M31 cannot use respiratory substrates such as ethanol to support growth. Strain M31, however, can be grown on complete glucose medium. As a fermentative substrate, glucose allows yeast cells to generate ATP through substrate-level phosphorylation.

Following transformation of the M31 host cells with the resultant recombinant plasmids, transformants were plated on solid selective glucose medium at $28^{\circ} \mathrm{C}$ for three days. In order to examine whether the allotopically expressed FLAG tagged-subunit 8 variants rescued the ethanol negative phenotype of strain M31, transformant colonies were transferred onto solid complete-ethanol-medium. Growth of transformants on the ethanol medium was observed after 4 days of incubation (Figure 1). Growth of FLAG taggedsubunit 8 variants on the ethanol medium indicated that the corresponding FLAG-tagged subunit 8 variant proteins had successfully assembled into functional enzyme complexes.

The present results showed that the three FLAG taggedsubunit 8 variants were assembled into functional enzyme complexes as indicated by the growth ability of the corresponding mutant strains (DF66, DF68, and DF71) on ethanol medium. The other three variants (DF67, DF69, and DF70) were found to be nonfunctional as indicated by the growth inability of the corresponding strains.

Basic Growth Characteristics of Strains Expressing FLAG Tagged-Subunit 8 Variants. The growth properties of the four strains expressing functional FLAG taggedsubunit 8 (a wild-type and three variants) were examined by determining their generation times for growth on liquidethanol-medium. Generation time or doubling time is the time needed for the population to double. The generation time (Table 2) was calculated from the growth curve of each strain. The generation time reflects the performance of the corresponding subunit 8 variant in the enzyme complex.

FLAG Tagged-Subunit-8 Variant Proteins Detected Using FLAG Monoclonal Antibody. Following mitochondrial isolation from strain FTC2, DF66, DF68, and DF71, an aliquot of $100 \mu \mathrm{g}$ mitochondrial protein of each variant was separated on SDS-PAGE. Mitochondrial proteins prepared from strain YM2 expressing subunit 8 protein without FLAG tags were included as controls. The proteins were then transferred to PVDF membrane. The membrane was cut into two portions. One portion of the membrane containing subunit 8 protein was probed with anti-FLAG M2 antibody as the primary antibody. The second portion of the membrane containing

Table 2 Generation times of strains expressing FLAG taggedsubunit 8 variants

$\begin{array}{lc}\text { Strain } & \text { Generation time }(\mathrm{h}) \\ \text { FTC2 } & 6.9 \pm 0.4 \\ \text { DF66 } & 8.2 \pm 0.5 \\ \text { DF68 } & 11.1 \pm 0.5 \\ \text { DF71 } & 7.2 \pm 0.5\end{array}$




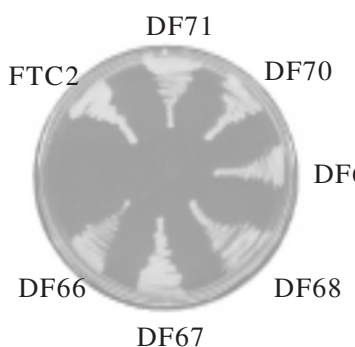

Glucose medium

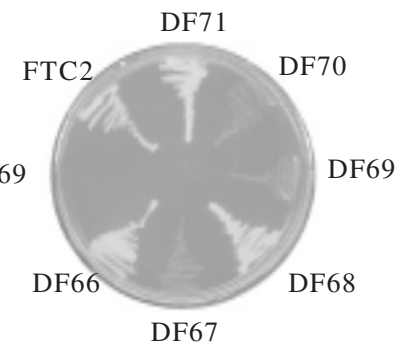

Ethanol medium
Figure 1 Functional assessment of allotopically expressed FLAG tagged-subunit 8 variants. Transformants (FTC2, DF66, DF67, DF68, DF69, DF70, DF71) were grown on solid selective glucose medium (left) and then transferred onto solid ethanol medium (right). The growth of the FLAG tagged-variants on ethanol medium indicated that the corresponding FLAG tagged-subunit 8 variant proteins are successfully imported into mitochondria upon their translation in the nucleocytosolic system, and are assembled into functional mitochondrial ATP synthase complexes.

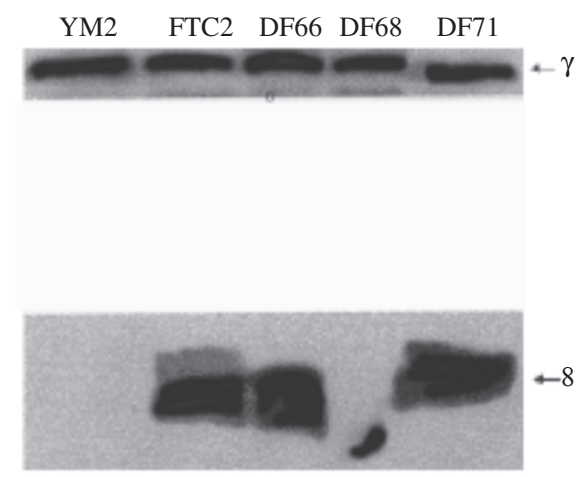

Figure 2 Detection of the FLAG tagged-proteins of subunit 8 variants. FLAG tagged-subunit 8 variants contained in mitochondrial lysates were detected in a Western blot analysis using the anti-FLAG M2 monoclonal antibody. Mitochondrial lysates prepared from a strain expressing wildtype subunit 8 without FLAG tag (YM2) were included as controls. FTC2 is strain expressing FLAG tagged-wildtype subunit 8. DF66, DF68, and DF71 are strains expressing FLAG taggedsubunit 8 variants. $\gamma=$ subunit $\gamma$ of yeast mitochondrial ATP synthase, $8=$ subunit 8 of yeast mitochondrial ATP synthase.

subunit $\gamma$ protein was probed with anti-subunit $\gamma$ antibody. Detection of subunit $\gamma$ was employed as a positive control. Results (Figure 2) showed that the FLAG tagged-subunit 8 variant proteins could be detected using anti-FLAG M2 monoclonal antibody. Subunit $\gamma$ was also detectable in each sample. As expected, the non-tagged-subunit 8 protein isolated from the YM2 strain was not detectable.

\section{DISCUSSION}

The present study attempted to elucidate the significant interaction of the CHD with the lipid bilayer of the inner mitochondrial membrane as well as to analyze the effects of addition of charges residues at the $\mathrm{C}$-terminus region of subunit 8 variants. In principal, the membrane spanning domain of subunit 8 should be disrupted upon the introduction of charged amino acids because the lipid bilayer is unable to accommodate unshielded charged residues. The present results show that among the functional FLAG tagged-subunit 8 variants, the variant DF68, which has double charged residues in the middle of the CHD, displays the most functional defects in ATP synthase activity as indicated by the slowest growth rate (Table 2) of this strain on ethanol medium. These observations support the previous data (Roucou et al. 1999) obtained from non-FLAG taggedsubunit 8 variants suggesting that the hydrophobic characteritics of residues 23 and 24 of subunit 8 is critical for maximal ATP synthase activity.

Subunit 8 is sensitive to manipulation of charged residues at the C-terminus (Grasso et al. 1991). From the present study it is clear that addition of the charged hexapeptide FLAG tag at the $\mathrm{C}$-terminus of subunit 8 variants affected the function of the FLAG tagged-variants. Three (DF67, DF69, DF70) out of six FLAG tagged-double charged variants failed to restore growth of M31 cells on ethanol (Figure 1). The highly charged FLAG tag changes the nature, number, and distribution of the charged residues along the $\mathrm{C}$-terminal region.

The FLAG tag has mostly negative charged residues. The failure of the DF70 variant to function, as measured by growth on ethanol (Figure 1), might be due to the concentration of unfavourable negative charges along the C-terminal region of the DF70 variant protein. It should be noted here that the distribution of the negatively charged residues within the CHD is critical. When the adjacent negatively charged residues are not too close to the Cterminus region (asp23, Asp24 in DF68), the FLAG residues seemed to be more tolerated as is indicated by the ability of the DF68 variant to assemble a functional ATP synthase complex, despite some deficiency in performance. Moreover, when the double negatively charged substitution is further away from the C-terminus region (Asp16, Asp17 in DF66), the FLAG residues are well tolerated. This is well reflected by the growth rate of DF66 which is faster than the growth rate of DF68, based on growth measured by generation time (Table 2). To this end, the present studies suggest that a concentration of negatively charged residues at the Cterminal region of subunit 8 has an adverse effect on subunit 8 . This may be due to the presence of unfavourable chargecharge interactions with the positively charged residues along this region.

The interaction of the adjacent positively charged residues with the residues of the FLAG tag seems to exhibit a gradient of opposite direction compared to that of the negatively charged residues. When the adjacent positively charged residues are closest to C-terminus ( $\operatorname{Arg} 29, \operatorname{Arg} 30$ in DF71) the FLAG residues were well tolerated as indicated by the relatively fast growth rate of DF71. When the double positively charged residues are further away from the Cterminus region (Arg 16, Arg 17 in DF67 and Arg23, Arg24 in DF69) the FLAG tag residues are not tolerated. It seems therefore that the presence of additional positively charged residues closest to the $\mathrm{C}$-terminus of subunit 8 is favourable to counterbalance the presence of negatively charged FLAG tag residues.

All of the FLAG tagged-subunit 8 variant proteins (DF66, DF68, DF71) can be detected in a Western blot analysis using anti-FLAG M2 monoclonal antibody. As shown in Figure 2, however, for the same amount of mitochondrial lysate analyzed, the signal detected for the DF68 lysate is much weaker. At this stage, it is not clear as to whether this particular variant protein undergoes proteolytic degradation or whether there is an assembly defect associated with this particular 
variant causing a lesser amount of subunit 8 to be present in the complex.

The FLAG-tagged variant protein of DF66 showed an altered mobility in that this variant protein moves slightly faster in SDS-PAGE compared to the wildtype. A similar alteration in mobility is also clearly shown by the mutant protein of DF68. It is possible therefore that this altered mobility is due to the presence of the negatively charged aspartate residues within the CHD of both DF66 and DF68.

\section{ACKNOWLEDGEMENTS}

This work was supported by the AusAID. I would like to thank Rodney J. Devenish and Phillip Nagley of the Department of Biochemistry and Molecular Biology, Monash University, for guidance and providing facilities.

\section{REFERENCES}

Artika IM. 2006. Allotopic expression of a gene encoding FLAG tagged-subunit 8 of yeast mitochondrial ATP synthase. Hayati 13:36-38.

Bradford M. 1976. A rapid sensitive method for the quantities of proteins utilising the principal of protein-dye binding. Anal Biochem 72:248-254.

Cox GB, Devenish RJ, Gibson F, Howitt SM, Nagley P. 1992. The structure and assembly of ATP synthase. In: Ernster L (ed). Molecular Mechanisms in Bioenergetics. Amsterdam: Elsevier. p 283-315.

Fronzes R, Weimann T, Vaillier J, Velours J, Brethes D. 2006. The peripheral stalk participates in the yeast ATP synthase dimerization independently of e and $\mathrm{g}$ subunits. Biochemestry 45:6715-6723

Gearing DP, McMullen GL, Nagley P. 1985. Chemical synthesis of a mitochondrial gene designed for expression in the yeast nucleus. Biochem Int 10:907-915.

Grasso DG, Nero D, Law RHP, Devenish RJ, Nagley P. 1991. The Cterminal positively charged region of subunit 8 of yeast mitochondrial ATP synthase is required for efficient assembly of this subunit into the membrane $\mathrm{F}_{0}$ sector. Eur J Biochem 199:203209.

Gray RE, Law RHP, Devenish RJ, Nagley P. 1996. Allotopic expression of mitochondrial ATP synthase genes in the nucleus of Saccharomyces cerevisiae. Methods Enzymol 264:369-389.

Klebe RJ, Harris JV, Sharp ZD, Douglas MG. 1983. A general method for chemically induced transformation of bacteria and yeast. Gene 25:333-341.

Laemmli UK. 1970. Cleavage of structural proteins during the assembly of the head of bacteriophage T4. Nature 227:680-685.

Lang BF et al. 1977. A simple method for the large-scale preparation of mitochondria from microorganism. Anal Biochem 77:110121

Law RHP, Devenish RJ. 1988. Expression in yeast of antisense RNA to ADE1 mRNA. Biochem Int 17:673-679.

Macreadie IG et al. 1983. Biogenesis of mitochondria: the mitochondrial gene (aap1) coding for mitochondrial ATPase subunit 8 in Saccharomyces cerevisiae. Nucl Acids Res 11:44354451.

Nagley $\mathrm{P}$ et al. 1988. Assembly of functional proton-translocating ATPase complex in yeast mitochondria with cytoplasmically synthesised subunit 8 , a polypeptide normally encoded within the organelle. Proc Natl Acad Sci USA 85:2091-2095.

Nagley P et al. 1990. Subunit 8 of yeast mitochondrial ATP synthase: biochemical genetics and membrane assembly. In: Kim $\mathrm{CH}$, Ozawa $\mathrm{T}$ (ed). Bioenergetics, Molecular Biology, Biochemistry and Pathology. New York: Plenum. p 305-325.

Papakonstantinou T, Law RHP, Nesbitt WS, Nagley P, Devenish RJ. 1996. Molecular genetic analysis of the central hydrophobic domain of subunit 8 of yeast mitochondrial ATP synthase. Curr Genet 30:12-18.

Roucou X, Artika IM, Devenish RJ, Nagley P. 1999. Bioenergetic and structural consequences of allotopic expression of subunit 8 of yeast mitochondrial ATP synthase. The hydrophobic character of residues 23 and 24 is essential for maximal activity and structural stability of the enzyme complex. Eur J Biochem 261:444-451.

Sambrook J, Fritsch EF, Maniatis T. 1989. Molecular Cloning - a Laboratory Manual. $2^{\text {nd }}$ edition. New York: Cold Spring Harbor.

Stephens AN, Roucou X, Artika IM, Devenish RJ, Nagley P. 2000. Topology and proximity relationships of yeast mitochondrial ATP synthase subunit 8 determined by unique introduced cysteine residues. Eur J Biochem 267:6443-6451. 
